Letter to the Editor

\title{
Impact of inertia on possible fundamental drawbacks in radiochromic film dosimetry
}

\section{History of radiochromic film dosimetry}

From its very inception [1] radiochromic films draw an immediate attention for its high spatial resolution and near tissue equivalence in most dosimetry applications [2]. The earlier GafChromic ${ }^{\mathrm{TM}}$ film models (MD-55, HS) were quite expensive, limiting their use to experimental dosimetry. The renaissance of radiochromic film dosimetry came about in 2005 with the introduction of the EBT film model [3] with significantly lower (10 times per square inch lower) price and readily accepted radiochromic film protocols (RFPs) using inexpensive flatbed document scanners [4]. To date, a number of RFPs have been described, using either transmission single [5] or multiple color channels [6], or reflection scanned images [7].

\section{Possible drawbacks}

Despite relatively steep development of radiochromic film dosimetry, the authors of this letter came across two significant errors in using radiochromic film dosimetry within not only reviewing phase but in the published articles in some very respectful journals. We sincerely believe the omissions in published papers came due to lack of focus by both authors and reviewers, and our intention is to simply bring to the attention of film users the two issues that may potentially lead to significant errors. The first issue is the use of non-linear response of radiochromic film (netOD, raw $P V$ ) and attributing a simple division of such response functions to relative dose. Second problem is the lack of awareness that radiochromic films exhibit a significant energy dependent response when irradiated in low energy photon beams. Such obvious, yet significant omissions could be eventually explained by inertia, as laid down by Newton, more than three centuries ago [8]. It seems that inertia does not only drive the mechanics of the Universe, but the human way of life too.

\section{Non-linearity of response function}

Radiographic film dosimetry was around for much longer than radiochromic film based one. At the time, radiographic film response to exposure was commonly expressed in terms of optical density $(O D)$, a quantity derived as logarithm for base 10 of inverse transmission. Rationale for choosing this quantity was the H\&D curve, which exhibited linear relation in relatively wide dose range, except at low (toe) and high (saturation) dose values. Quite possibly, the radiochromic film user's community followed (by inertia) the suit in using the very same quantity despite the fact the response of radiochromic film was non-linear in the whole dose range [9]. We can only make a hypothesis that some users tend to use ratio of optical densities (for measuring either $P D D$ curves or profiles) following the practice (inertia) with ion-chambers, who (again in certain ranges of beam qualities) have linear response (measured charge) with dose. When the same methodology is used for (any) dosimeter having non-linear response, such obtained "relative values" in the case of radiochromic films will overestimate the real relative dose values [10]. Over the last decade, a few novel functional forms have been suggested that do linearize the response of radiochromic films $[10,11]$. However, one has to be cautious with using even these new functional forms, as they would provide accurate relative dose values by simple division of responses if and only if the film response for the given beam quality does not change over the plane in which film is used for relative dose measurements.

\section{Radiochromic film response as a function of beam quality}

The second possible drawback that may lead to inaccurate dose measurements is if one ignores the energy dependent response at low photon energies. It was well established that all the EBT film models exhibit strong energy dependence as the effective beam energy goes bellow $100 \mathrm{keV}$ [12-14]. Nevertheless, one may find papers measuring relative dose distributions with films at $50 \mathrm{kVp}$ beam quality and reporting relative dose by completely ignoring this effect by using calibration curve obtained at either different $\mathrm{kVp}$ beam quality or sometimes even using calibration at MV beam. We believe this might be yet another case of inertia, following the widely adopted prejudice that film has energy independent response based on the fact that most of the early research with films has been performed in megavoltage photon and electron beams, the range of beam qualities where radiochromic film indeed has response to dose independent of energy [15].

How to avoid the two drawbacks when performing radiochromic film dosimetry

When measuring the relative dose, user could use any function that make the film response linear with dose. This can be easily done once calibration films are scanned - data is there forever to experiment with. If, for whatever reason (existing software, existing protocols), user prefers to use response functions with non-linear response (netOD, raw $P V$ ), to obtain relative dose distribution, one has to go through the reference dosimetry protocol, where the calibration curve function will sort out the non-linear response. Once the issue with response function is solved, user has to make sure dose distribution is measured for photon beam qualities with effective energy above $100 \mathrm{keV}$. For lower photon energies, one has to determine beam quality at every measurement point within the film plane, and then apply the corresponding calibration curve for each measurement point. For this task, user clearly has to go through the reference dosimetry protocol for each measurement point irrespectively of using response function with linear or nonlinear response. 


\section{References}

[1] Chu RDH, VanDyke G, Lewis DF, O'Hara KPJ, Buckland BR, Dinelle F, GafChromic dosimetry media: a new high dose rate thin film routine dosimeter and dose mapping tool. Radiat Phys Chem 1990;35:767-73.

[2] Devic S. Radiochromic film dosimetry: past, present, and future. Phys Med 2011;27:122-34.

[3] Cheung T, Butson MJ, Yu PK. Post-irradiation colouration of Gafchromic EBT radiochromic film. Phys Med Biol 2005;50:N281-5.

[4] Devic S, Seuntjens J, Hegyi G, Podgorsak EB, Soares CG, Kirov AS, et al. Dosimetric properties of improved GafChromic films for seven different digitizers. Med Phys 2004;31:2392-401.

[5] Devic S, Seuntjens J, Sham E, Podgorsak EB, Kirov AS, Schmidtlein RC, et al. Precise radiochromic film dosimetry using a flat-bed document scanner. Med Phys 2005;32:2245-53.

[6] Micke A, Lewis DF, Yu X. Multichannel film dosimetry with nonuniformity correction. Med Phys 2011;38:2523-34.

[7] Alva H, Mercado-Uribe H, Rodriguez-Villafuertre M, Brandan ME. The use of a reflective scanner to study radiochromic film response. Phys Med Biol 2002; 47:2925-33.

[8] Newton I. The Principia: mathematical principles of natural philosophy. Translated by: Cohen IB, Whitman AM. Berkeley, Calif.; London: University of California Press, 1999.

[9] Devic S, Tomic N, Lewis D. Reference radiochromic film dosimetry: review of technical aspects. Phys Med 2016;32:541-56.

[10] Devic S, Tomic N, Aldelaijan S, DeBlois F, Seuntjens J, Chan MF, et al. Linearization of dose-response curve of the radi-ochromic film dosimetry system. Med Phys 2012;39:4850-7.

[11] Aldelaijan S, Devic S. Comparison of dose response functions for EBT3 model GafChromic (TM) film dosimetry system. Phys Med 2018;49:121-6.

[12] Sutherland JGH, Rogers DWO. Monte Carlo calculated absorbed-dose energy dependence of EBT and EBT2 film. Med Phys 2010;37:1110-6.
[13] Chiu-Tsao S, Massillon JLG, Domingo-Muñoz I, Chan M. Energy dependence of the new GafChromic-EBT3 film's dose response-curve. Med Phys 2012;39(6Part11):3724.

[14] Bekerat H, Devic S, DeBlois F, Singh K, Sarfehnia A, Seuntjens J, et al. Improving the energy response of external beam therapy (EBT) GafChromic ${ }^{\mathrm{TM}}$ dosimetry films at low energies ( $\leq 100 \mathrm{keV}$ ). Med Phys 2014;41:022101.

[15] Sarfehnia A, Stewart K, Seuntjens J. An absorbed dose to water standard for HDR ${ }^{192}$ Ir brachytherapy sources based on water calorimetry: numerical and experimental proof-of-principle. Med Phys 2007;34:4957-61.

Slobodan Devic

Medical Physics Unit, McGill University, Montréal, Québec, Canada Department of Radiation Oncology, Jewish General Hospital, Montréal, Québec, Canada

Segal Cancer Centre, Jewish General Hospital, McGill University, Montréal, Québec, Canada E-mail address: slobodan.devic@mcgill.ca.

Saad Aldelaijan

Medical Physics Unit, McGill University, Canada

Biological \& Biomedical Engineering Department, Montreal Neurological Institute, Montréal, Québec, Canada Biomedical Physics Department, King Faisal Specialist Hospital \& Research Centre, Riyadh, Saudi Arabia

Hamed Bekerat

Medical Physics Unit, McGill University, Montréal, Québec, Canada Department of Radiation Oncology, Jewish General Hospital, Canada

*Address: Jewish General Hospital, 3755 chemin de la Côte-Sainte-Catherine, Montréal, Québec H3T 1E2, Canada. 